\title{
A MARKOV MODEL TO PREDICT WEAR STATUS OF THE VEHICLE TYRES
}

\author{
Alina Pricopie ${ }^{1}$, Laurențiu Frangu ${ }^{1}$, Sergiu Caraman ${ }^{1}$ \\ "Dunărea de Jos" University of Galati, Department of Automatic Control and Electrical Engineering, \\ Domnească Street, 47, 800008, Galati, Romania
}

\begin{abstract}
The paper deals with the integration of the wear process into a statistical model of the status of vehicle tyres. The probabilities of the states are provided by a Markov model. The considered phenomena are the accidental perforation of the tyre, the repair of the perforated tyre and the wear, meaning the loss of rubber material on the tyre surface. The model predicts the probability of exceeding the limit of the safe operating, for a batch of tyres. It is useful to determine the optimum time for the replacement of the tyres. Supplementary, periodic identification of the model parameters allows the setting of an alarm about unusual phenomena that affect the safe operation. More versions of the statistical distributions of the parameters are studied. The results are validated by numerical simulation.
\end{abstract}

Keywords: tyre wear, Markov model, probability, statistical distribution

\section{INTRODUCTION}

Due to its multiple economic and environmental implications, the tyre wear has been an intensely researched subject (Braghin, et al., 2006), (Veen, 2007), (Salminen, 2014), (Zuo, 2011). Usually, for manufacturer studies, the wear is measured on the tyre, for a short sampling period (Lukper, et al., 2002), (Koike, 2002). Many phenomena that cause wear are analyzed separately. A detailed description of the laboratory tests performed over time and the aspects they followed and founded are presented in (Klueppel, 2014). In this paper we want to anticipate the instant when the tyre operating becomes unsafe and to detect the possible unexpected wear phenomena, for lots of tyres. This action reflects the perspective of the user (a transport company, for example) who wants to obtain a prediction with few measurements.

For this purpose, the frequent measurement of the wear is not practical. Simplified wear models and a Markov model to estimate the probabilities of tyre conditions are used. The causes leading to the variations of the parameters of the simplified model (poor quality of the roads surface, aggressive behavior of the driver, errors in the mechanical adjustments etc.) are not considered separately, but their existence is detected from the anticipated probabilities of the Markov model. A collective of researchers used Markov models with a large number of states (Tananaev, 2014), which is not suitable for our purpose (they are too complex). A simple Markov model was used to anticipate the state of wear of the valves in the sugar industry by Pricopie, et al., (2020). The degradation model described in the previously mentioned paper is used, in the conditions in which the Markov model was extended to include the tyre repair and the wear rate was considered a random variable.

Further, in Section 2, the simplified models of wear with various versions of probability density functions are presented. Section 3 presents the Markov model. The meanings of the probabilities provided by this 
model and the experimental results obtained by numerical simulations are presented in Section 4. Finally, Section 5 is dedicated to the paper conclusions.

\section{THE WEAR MODELLING}

There are two main processes that influence the status of the tyre which are considered in this paper: the wear and the perforation of the tyre. Both are degradation processes. There are two purposes of the evaluation of these processes:

- the prediction of the service time, before the wear exceeds the threshold admitted by the law;

- the detection of unexpected persistent phenomena which influence the safety and the costs.

The wear consists in the loss of rubber from the tyre tread (the part of the tyre which rolls on the road). It has been extensively studied by the tyre producers and it involves more sub-processes (Salminen, 2014, Kluppel, 2014): adhesion wear, abrasive wear, hysteresis, friction etc. The advance of the wear is influenced by many factors: the quality of the tyre, quality of the road's pavement, driving style, mechanical settings of wheel and suspension, weight of the vehicle etc. In this work, only the overall statistical results of these processes are considered. It is expressed by the depth of the tyre tread. The safety of the traffic implies that worn tyres are not allowed to run on public roads, as soon as the depth decreased under a specified threshold.

The perforation of the tyre is the second process considered in the paper ("flat tyre"). Usually, it produces economic rather than safety consequences. After a perforation, the tyre can be repaired and further used, but frequent events suggest a persistent cause, such as poor quality of the tyre or of the road. This process is characterized by the record of the instants when the tyre suffered perforations. The tyre may also be severely destroyed, during accidents, when the tyre material is ripped. However, this phenomenon is not frequent, and thus, it was neglected in the present work.

The wear is expressed by a monotonously increasing function, $u z(s)$. The function is dimensionless. Its values span from 0 to 1 , where the value 0 means a brand new tyre and means a flat profile of the tread or 0 value of its depth. The model of the wear process is (1):

$$
\text { (1) } u z(s)=v \cdot s
$$

where $v$ represents the wear rate. It was adapted from Pricopie, et al., (2020), with a change in the nature of the variable. For practical reasons, the independent variable of this model, denoted by " $s$ " is the distance covered by the tyre, expressed in $\mathrm{km}$. The wear rate
" $v$ " is a random variable, whose value depends on many statistical factors, which is not detailed in this paper. The dimension of the wear rate is $\mathrm{km}^{-1}$. According to the model (1), the wear function evolves as in Fig. 1, for a fixed value of the wear rate, $v=1 / 50,000 \mathrm{~km}^{-1}$.

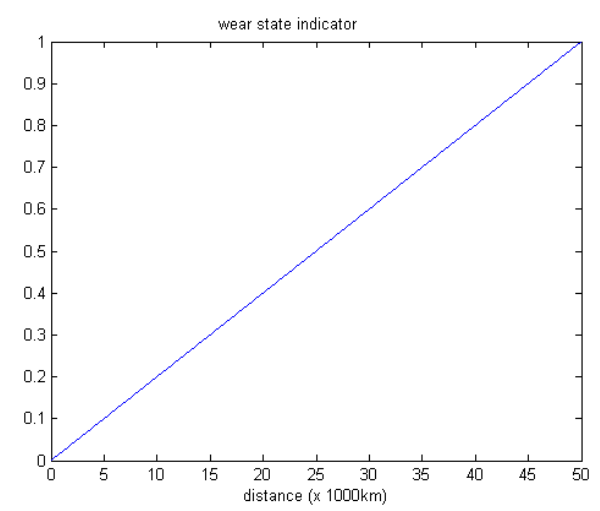

Fig. 1. Evolution of the wear, for a fixed value of the wear rate

The phenomenon of tyre perforation is described by a statistical model (2), adopted from (Pricopie, et al., 2020), too:

$$
\text { (2) } P=d \cdot \Delta s
$$

where $P$ stands for the probability of the perforation, within the infinitely small interval, $\Delta \mathrm{s}$. The perforation rate " $d$ " is also a random variable whose dimension is $\mathrm{km}^{-1}$. Fig. 2 present the average number of perforation events for a fixed value of the perforation rate, $d=1 / 12,000 \mathrm{~km}^{-1}$.

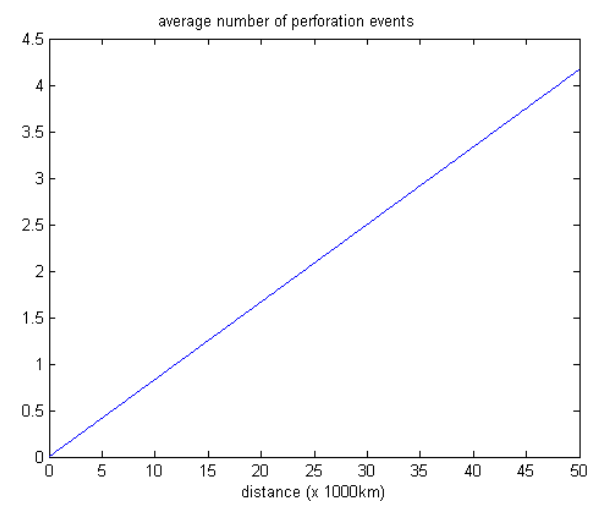

Fig. 2. Evolution of the average number of perforation events, for a fixed value of the perforation rate

During the last part of the tyre life the wear and the perforation rates seem to be connected parameters, because the perforation is more probable when the tyre is severely worn. However, the goal of this paper is to anticipate the state probabilities, up to the state 2 , so the model considers the two rates as independent random variables. 
In order to use these simple models to predict the wear and the reliability of the tyres, the probability density functions of the parameters should be known. Three distribution models were adopted in this paper for the probability density functions of the parameters: uniform, triangular and normal (gaussian). Fig. 3 presents the shapes of these three distributions. The values of the wear rate span on the horizontal axis, whereas the probability density is represented on the vertical axis. All versions observe the restriction that the probability density function integrates to 1 . The mean of the probability density function was chosen the same, in all three cases: $v=$ $1 / 50,000 \mathrm{~km}^{-1}$. The interval of nonzero density, for the first two versions, spans from $0.4 \cdot v$ to $1.6 \cdot v$. The third version (normal distribution) is characterized by the standard deviation which was chosen $0.2 \cdot v$.

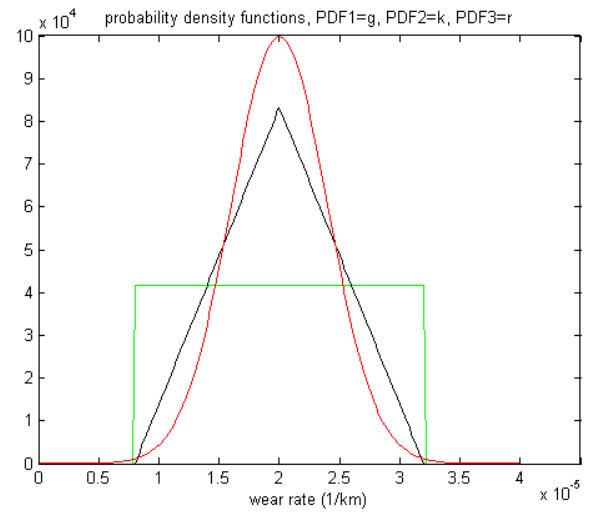

Fig. 3. Three versions of the probability density function of the wear rate: uniform, triangular, normal

The deviation of the statistical parameters from the usual known values raises an alarm about unexpected persistent phenomena during the exploitation of the tyres.

The evaluation of the probabilities of the tyre states, described in the next section, requires the values of the probabilities:

$$
\text { (3) } P(v<t h)=\int_{0}^{t h} p_{v}(x) d x \text {, }
$$

where $p_{v}(x)$ is the probability density function of the parameter $v$.

\section{THE MARKOV MODEL}

The Markov model is useful for computing the probabilities of the different states of the tyre. These probabilities are further used for choosing the most suitable replacing strategy. The considered states of the tyre are the following:

State 0 (new or almost new valid tyre) when the wear is not yet a threat for the safety. The possible transitions from state 0 are to state 1 , through perforation and to state 2 , through wear.
State 1 (perforated tyre): the transition to this state is a random discrete event. It is possible to reach this state from state 0 and state 2 . The action of repairing the tyre is performed fast enough and the distance covered in this state is 0 . The transition from this state is always back, to the state indicated by the wear. The condition of the tyre, after repairing it, is "as bad as old".

State 2 (worn tyre) when the wear indicator exceeds the threshold allowed by law. In terms of measurements, that means the depth of the tread profile is too low. For simplicity, a fixed value of the threshold is considered, although it depends on the tyre geometry and on the national laws. The value of the threshold of the wear indicator was chosen $R=$ $60 \%$.

State 3 (defective tyre) when the wear indicator reached the level of $100 \%$ or beyond.

The diagram of the Markov model is presented in Fig. 4.

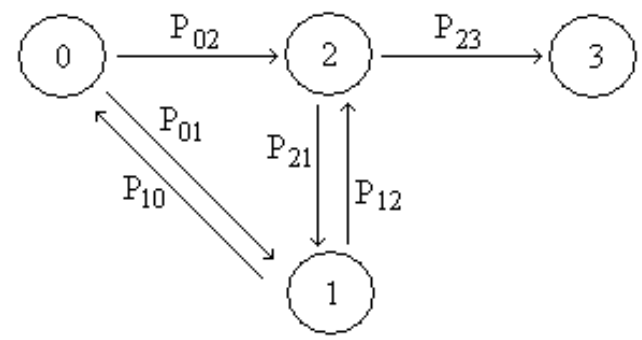

Fig. 4. Diagram of the Markov model

According to the objective of this paper, the probability of state 3 is not interesting, because the tyre has to be replaced, as soon as it reaches state 2 . However, all states have to be considered in order to build a systematic model. The outcome of the model is the set of probability functions associated with each state. The required parameters are the transition probabilities, associated to each arch of the model. In Fig. 3 only the relevant transition probabilities were represented. For practical reasons, a model with discrete values of the variable was used, i.e. a discrete covered distance. The sampling period is $\Delta \mathrm{s}=50 \mathrm{~km}$, and the covered distance is $s=k \cdot \Delta s$. For simplicity, the states are considered not to change during the sampling period. According to the degradation models, presented in the previous section, the relevant transition probabilities are described by (4)-(9). They refer to the transitions during the sampling period, at the discrete distance $\mathrm{k}$.

$$
\text { (4) } P_{01}(k)=d \cdot \Delta s
$$

$$
\text { (5) } \begin{gathered}
P_{02}(k)=P(u z(k)>R)=P(v \cdot k \cdot \Delta s>R)= \\
P\left(v>\frac{R}{k \cdot \Delta s}\right)
\end{gathered}
$$


(6) $P_{10}(k)=P(u z(k) \leq R)=P(v \cdot k \cdot \Delta s \leq R)=$ $P\left(v \leq \frac{R}{k \cdot \Delta s}\right)=1-P_{02}(k)$

(7) $P_{12}(k)=P_{02}(k)$

(8) $P_{21}(k)=P_{01}(k)$

(9) $P_{23}(k)=P(u z(k) \geq 1)=P\left(v>\frac{1}{k \cdot \Delta s}\right)$

In eqs. (5), (6), (9) the probabilities are computed as indicated in (3). The probabilities $P_{03}, P_{11}, P_{13}, P_{20}$, $P_{30}, P_{31}, P_{32}$ and $\mathrm{P}_{33}$ are null, as they are associated with not possible transitions. The rest of the transition probabilities are derived from those above:

$$
\begin{aligned}
& \text { (10) } P_{00}(k)=1-P_{01}(k)-P_{02}(k) \\
& \text { (11) } P_{22}(k)=1-P_{21}(k)-P_{23}(k)
\end{aligned}
$$

The integration of the model (4) - (11) is straightforward, by a recursive procedure. One can notice that the probability of state 1 is not relevant (tends towards 0), as we considered that the perforated tyre is repaired with no delay. However, the number of transitions in state 1 is relevant, it provides the average number of perforations. The initial values are: $P_{0}(0)=1, P_{1}(0)=P_{2}(0)=$ $P_{3}(0)=0$. The integrating procedure follows the equations (12) - (14):

$$
\begin{gathered}
\text { (12) } \begin{array}{c}
P_{0}(k)=P_{0}(k-1) \cdot\left(1-P_{01}(k)-P_{02}(k)\right)+ \\
P_{1}(k-1) \cdot P_{10}(k)
\end{array} \\
\text { or } P_{0}(k)=P_{0}(k-1) \cdot\left(1-P_{02}(k)\right) \\
\text { (13) } P_{2}(k)=P_{2}(k-1) \cdot\left(1-P_{21}(k)-P_{23}(k)\right)+ \\
P_{1}(k-1) \cdot P_{12}(k)+P_{0}(k-1) \cdot P_{02}(k) \\
\text { or } P_{2}(k)=P_{2}(k-1) \cdot\left(1-P_{23}(k)+P_{0}(k-1) \cdot\right. \\
P_{02}(k)
\end{gathered}
$$$$
\text { (14) } P_{3}(k)=P_{3}(k-1)+P_{2}(k-1) \cdot P_{23}(k)
$$

In eqs. (12) and (13) the terms containing $P_{1}(k-1)$ were removed, because the tyre regains the state prior to the perforation, after repair. That means that $P_{0}(k-1) \cdot P_{01}(k)=P_{1}(k-1) \cdot P_{10}(k) \quad$ and $P_{2}(k-1) \cdot P_{21}(k)=P_{1}(k-1) \cdot P_{12}(k)$.

The solution (12) - (14) provides the expected state probabilities. The main issue when integrating the model is the random nature of the parameters $v$ and $d$, as presented in the previous section. The information we can use to confirm or not the model's prediction are: the tread depth (reflecting the loss of rubber) and the sequence of the instants when the tyre was perforated.

\section{SIMULATION RESULTS}

As previously mentioned, the parameters of the degradation model are independent. Additionally, they have effect on two different measurable variables - the tyre tread depth and the number of perforations. Consequently, their influence was studied separately. The evolution of the average number of perforation events was presented in Fig. 2. If a deviation appears, one can suspect an internal cause (poor quality of the tyre material) or an external one (poor quality of the road or improper driving style).

The influence of the wear rate on the tyre tread depth is more diversified. Fig. 5 presents the evolution of the wear indicator, $u z(s)$, for two different values of the parameter. It it obvious that the effects of these two values are quite different, in terms of travelled distance, up to the allowed threshold. The fast wear is associated with bad tyre, bad roads, improper driving style or wrong mechanical settings of the vehicle.

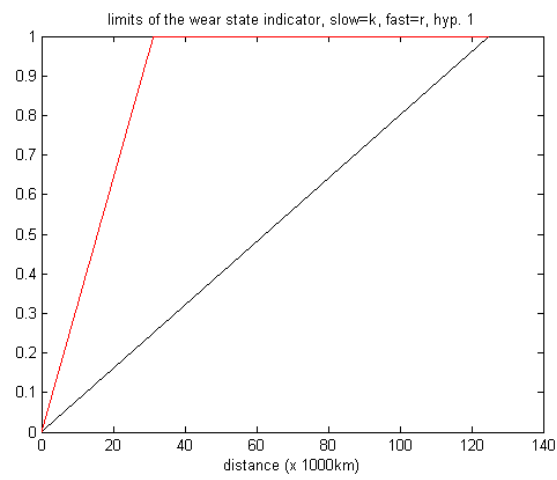

Fig. 5. Evolution of the wear indicator, for the extreme values of the wear rate (uniform and triangular distributions, Fig. 3)

The evolution of the transition probabilities has the aspect in Fig. 6 or similar. The aspect of the state probabilities is presented in Figs. 7, 8, 9, for the three considered distributions of the wear rate.

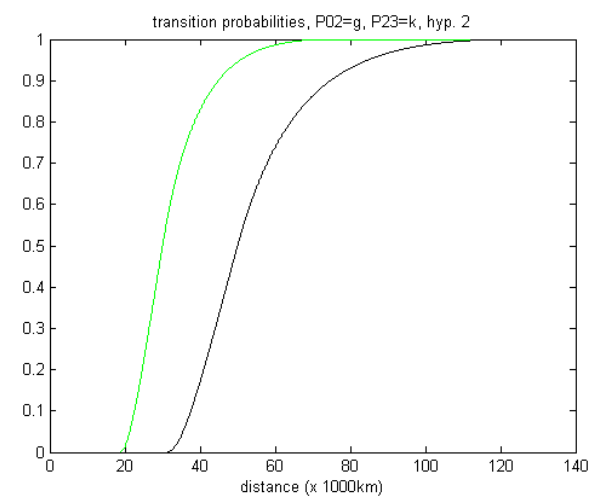

Fig. 6. Transition probabilities $\left(P_{02}, P_{23}\right)$ for the triangular distribution 
THE ANNALS OF "DUNĂREA DE JOS" UNIVERSITY OF GALATI

FASCICLE III, 2020, VOL. 43, NO. 1, ISSN 2344-4738, ISSN-L 1221-454X

state probabilities, $\mathrm{PSO}=\mathrm{g}, \mathrm{PS} 2=\mathrm{k}, \mathrm{PS} 3=\mathrm{r}$, hyp. 1

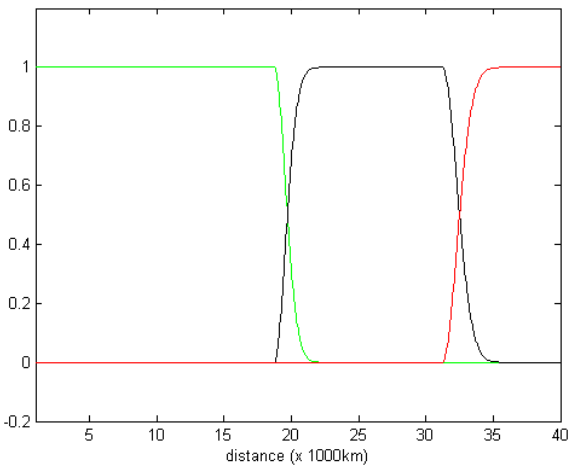

Fig. 7. State probabilities, uniform distribution

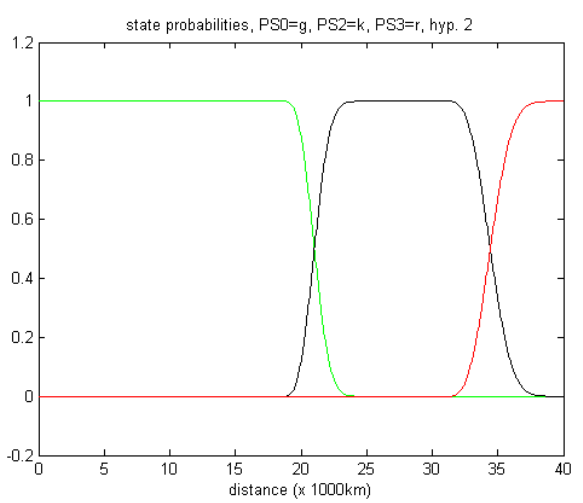

Fig. 8: state probabilities, triangular distribution

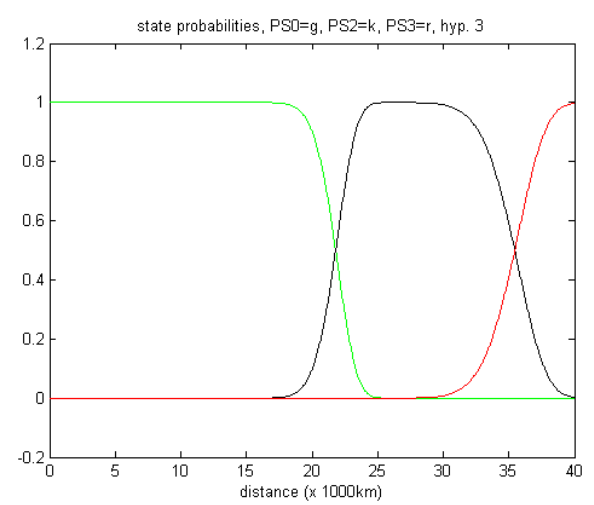

Fig. 9: state probabilities, normal (gaussian) distribution

When comparing these three results (Figs. 7, 8, 9), one can notice the minor difference between them. That means that the chosen distribution (shape of the probability density function) does not significantly modify the expected travelled distance, up to the limit of the tyre tread depth (state 2). For instance, state 2 is reached somewhere between $20-22,000 \mathrm{~km}$, in the three diagrams. The stronger influence is exerted by the mean of this distribution, as it is visible in Fig. 5. Similar to the case of the other parameter, periodic examination of the tread depth can indicate unexpected external or internal causes that can shorten the tyre's service life. For instance, this examination can take place every 1000 or 2000 $\mathrm{km}$.

\section{CONCLUSIONS}

The model allows the prediction of the service life, i.e. the distance travelled up to the wear limit, which allows safe exploitation. The shape of the probability density function proved to have low influence on the predicted service life, whereas the mean of the distribution has a strong influence. For a set of tyres, the deviation of the estimated parameters from the known intervals can be used for the detection of unexpected wear processes, which have to be further investigated.

\section{ACKNOWLEDGEMENT}

This work is supported by the project ANTREPRENORDOC, in the framework of Human Resources Development Operational Programme 2014-2020, financed from the European Social Fund under the contract number 36355/23.05.2019 HRD OP /380/6/13 -SMIS Code: 123847

\section{REFERENCES}

Tananaev D. et al. (2014). Identification of Exploitation Conditions of the Automobile Tyre while Car Driving by Means of Hidden Markov Models, Young Scientists' International Workshop on Trends in Information Processing.

Salminen H., (2014). Parametrizing tyre wear using a brush tyre model. Master thesis. Royal Institute of Technology Stockholm, Sweden.

Lupker H.A. and al. (2002). Truck tyre wear assessment and prediction, 7th International Symposium on Heavy Vehicle Weights \& Dimensions Delft, The Netherlands, June 16-20.

Koike A. et all, A new type of tyre tester. Evaluation of tread wear resistance by laboratory testing, International Polymer Science and Technology, Vol. 29, No. 5, 2002.

Li Y, Zuo SG, Lei L, et al. Analysis of impact factors of tyre wear. J Vib Control 2011; 18: 833-840.

Veen J. An analytical approach to dynamic irregular tyre wear. Master's Thesis, Technische Universiteit Eindhoven, 2007.

Braghin F, Cheli F, Melzi S, et al. Tyre wear model: validation and sensitivity nalysis. Meccanica 2006; 41:143-156.

Pricopie A., Frangu L., Vilanova R. and Sergiu C. (2020). A preventive maintenance strategy for an actuator using Markov models, IFAC, Berlin

Klueppel, M., (2014). Wear and Abrasion of Tyres.In: Encyclopedia of Polymeric Nanomaterials, Kobayashi S., Müllen K. Springer, Berlin, Heidelberg. 